\title{
Combined Serum Creatinine and Cystatin C Schwartz Formula Predicts Kidney Function Better than the Combined CKD-EPI Formula in Children
}

\author{
H. Chehade ${ }^{a} \quad$ F. Cachat ${ }^{a} \quad$ A.-S. Jannot ${ }^{d} \quad$ B.-J. Meyrat ${ }^{b} \quad$ D. Mosig ${ }^{a} \quad$ D. Bardy ${ }^{c}$ \\ P. Parvex ${ }^{\mathrm{e}}$ E. Girardin ${ }^{\mathrm{a}}$
}

${ }^{a}$ Divisions of Pediatric Nephrology and ${ }^{b}$ Pediatric Surgery, Department of Pediatrics, ${ }^{c}$ Central Chemistry Laboratory, Lausanne University Hospital, Lausanne, ${ }^{d}$ Division of Clinical-Epidemiology, Department of Health and Community Medicine, and e Division of Pediatric Nephrology, Department of Pediatrics, University Hospitals of Geneva, Geneva, Switzerland

\section{Key Words}

Adult . Chronic kidney disease $\cdot$ Child · Glomerular filtration rate

\begin{abstract}
Background: The combined serum creatinine (SCreat) and cystatin C (CysC) CKD-EPI formula constitutes a new advance for glomerular filtration rate (GFR) estimation in adults. Using inulin clearances (iGFRs), the revised SCreat and the combined Schwartz formulas, this study aims to evaluate the applicability of the combined CKD-EPI formula in children. Method: 201 iGFRs for 201 children were analyzed and divided by chronic kidney disease (CKD) stages (iGFRs $\geq 90 \mathrm{ml} /$ $\mathrm{min} / 1.73 \mathrm{~m}^{2}, 90>$ iGFRs $>60$, and iGFRs $\leq 59$ ), and by age groups ( $<10,10-15$, and $>15$ years). Medians with $95 \%$ confidence intervals of bias, precision, and accuracies within $30 \%$ of the iGFRs, for all three formulas, were compared using the Wilcoxon signed-rank test. Results: For the entire cohort and for all CKD and age groups, medians of bias for the CKD-EPI formula were significantly higher $(p<0.001)$ and precision was significantly lower than the solely SCreat and the combined SCreat and CysC Schwartz formulas. We also found that using the CKD-EPI formula, bias decreased and accuracy increased while the child age group increased, with
\end{abstract}

\section{KARGER}

E-Mail karger@karger.com

www.karger.com/ajn a better formula performance above 15 years of age. However, the CKD-EPI formula accuracy is $58 \%$ compared to 93 and $92 \%$ for the SCreat and combined Schwartz formulas in this adolescent group. Conclusions: The performance of the combined CKD-EPI formula improves in adolescence compared with younger ages. Nevertheless, the CKD-EPI formula performs more poorly than the SCreat and the combined Schwartz formula in pediatric population.

(c) 2013 S. Karger AG, Basel

\section{Introduction}

Glomerular filtration rate (GFR) determination is important in daily medical care practice [1]. Several equations for estimating GFR (eGFR) were developed over the last decade [2] with varying degrees of accuracy across all GFR levels and ages [3, 4]. Recently, new serum creatinine (SCreat) and/or cystatin C (CysC)-based formulas were developed, both in adult [5-7] and pediatric populations [8-10], taking into consideration the change in the SCreat measurements method and the emerging $\mathrm{Cys} \mathrm{C}$ as an endogenous filtration marker for renal function assessment. The most widely used formula in adults and in children are the Chronic Kidney Disease Epidemi- 
Table 1. Characteristics and classification of patients' renal disorders

\begin{tabular}{lrrl}
\hline & $\begin{array}{c}\text { CKD } \\
\text { stage I }\end{array}$ & $\begin{array}{l}\text { CKD } \\
\text { stage II }\end{array}$ & $\begin{array}{l}\text { CKD stages } \\
\text { III-V }\end{array}$ \\
\hline Obstructive or reflux uropathy & 55 & 41 & 6 \\
Congenital and acquired single kidney & 21 & 17 & 5 \\
Polycystic kidney disease & 6 & 7 & 3 \\
Glomerulopathies & 6 & 8 & 1 \\
Hemolytic uremic syndrome & 3 & 3 & 2 \\
Post-chemotherapy & 0 & 3 & 1 \\
Other & 1 & 8 & 4 \\
\hline Total & 92 & 87 & 22 \\
\hline
\end{tabular}

CKD stage I, stage II, and stages III-V denote iGFRs $\geq 90,60-89$, and $\leq 59 \mathrm{ml} / \mathrm{min} / 1.73 \mathrm{~m}^{2}$, respectively.

ology Collaboration (CKD-EPI) [6] and the Schwartz [8] formulas, respectively. These formulas were developed in a selective population, and deriving a universal formula applicable for both population groups remains challenging. Using the gold standard method for measuring GFR, i.e. inulin clearance (iGFR), this study aims to evaluate the applicability of the new combined SCreat- and CysCbased CKD-EPI formula [7] in children across all CKD groups. It also aims to determine whether this formula presents a better performance in defined child age groups and in consequence can be applicable in infants and/or adolescents. To our knowledge, this is the first study evaluating the combined CKD-EPI formula in children.

\section{Material and Method}

\section{Population}

201 inulin clearances of 201 children aged between 3 and 18 years, all white except 2 black, with various chronic kidney disease (CKD) were analyzed. Our study population was divided into three groups: group 1 (CKD stage I) with iGFRs $\geq 90 \mathrm{ml} / \mathrm{min} /$ $1.73 \mathrm{~m}^{2}$, group 2 (CKD stage II) with iGFRs between 60 and $89 \mathrm{ml} /$ $\mathrm{min} / 1.73 \mathrm{~m}^{2}$, and group 3 (CKD stages III-V) with iGFRs $<60 \mathrm{ml} /$ $\mathrm{min} / 1.73 \mathrm{~m}^{2}$. We also divided the study population into three age groups ( $<10$ years, $10-15$, and $>15$ years). Demographic characteristics for the whole sample and by stages of CKD are summarized in table 1 . This study was approved by the local research ethics board and conducted in accordance with the ethical standards of the Helsinki Declaration.

Analytical Analysis

SCreat Calibration

SCreat was measured using the kinetic colorimetric compensated Jaffe method, which is closely aligned with the enzy- matic assay and standardized against the reference IDMS method. This technique has been validated in our central chemistry laboratory comparing the compensated Jaffe technique and the enzymatic method with an average difference of 0.99 $\mu \mathrm{mol} / \mathrm{l}$ and the $95 \%$ confidence interval (CI) of -6.586 to 8.566 $\mu \mathrm{mol} / \mathrm{l}$.

\section{CysC Calibration}

CysC was measured by particle-enhanced nephelometric immunoassay (PENIA) on BN ProSpec analyser (Siemens Healthcare Diagnostics). The results were multiplied by 1.174 as indicated in the Siemens customer bulletin to adjust to the values obtained with the assay standardized to the new traceable International Reference Preparation (IRP) - ERM ${ }^{\circledR}$-DA471/IFCC as recommended by the KDIGO (Kidney Disease Improving Global Outcomes) [11]. The normal reference range of $\mathrm{CysC}$ was $0.55-1.06 \mathrm{mg} / \mathrm{l}$. The inter-assay coefficients of variation (CVs) were $4.95 \%$ at $1.13 \mathrm{mg} / \mathrm{l}$ and $3.30 \%$ at $4.91 \mathrm{mg} / \mathrm{l}$. The intra-assay CVs were $2.0 \%$ at $1.71 \mathrm{mg} / \mathrm{l}$ and $2.3 \%$ at $5.37 \mathrm{mg} / \mathrm{l}$. Total measurement imprecision was 0.097 at $1.14 \mathrm{mg} / \mathrm{l}$.

\section{Glomerular Filtration Rate Estimation}

The eGFR was calculated according to the revised Schwartz formula [8], the combined SCreat and CysC Schwartz formula [10], and the combined CKD-EPI formula [7], and expressed in milliliters per minute per $1.73 \mathrm{~m}^{2}$ as follows:

- For the revised Schwartz formula:

$\mathrm{eGFR}=0.413 \times($ Height $/$ SCreat $)$

SCreat is expressed in milligrams per deciliter and height $(\mathrm{Ht})$ in $\mathrm{cm}$.

- For the combined Schwartz formula:

$$
\mathrm{eGFR}=39.8 \times(\mathrm{Ht} / \mathrm{SCreat})^{0.456} \times(1.8 / \mathrm{CysC})^{0.418} \times(30 / \mathrm{BUN})^{0.079}
$$
$\times(\mathrm{Ht} / 1.4)^{0.179}[\times 1.076$ if females $]$.

SCreat is expressed in milligrams per deciliter, CysC in milligrams per liter, $\mathrm{Ht}$ in $\mathrm{cm}$, and blood urea nitrogen (BUN) in milligrams per deciliter.

- For the combined CKD-EPI formula:

In females:

$\mathrm{eGFR}=130 \times(\text { SCreat } / 0.7)^{\mathrm{a}} \times(\text { CysC/0.8 })^{\mathrm{b}} \times 0.995^{\text {Age }}[\times 1.08$ if black]

(if SCreat $\leq 0.7$ and CysC $\leq 0.8: \mathrm{a}=-0.248$ and $\mathrm{b}=-0.375$;

if SCreat $\leq 0.7$ and CysC >0.8: $a=-0.248$ and $b=-0.711$;

if SCreat >0.7 and CysC $\leq 0.8: \mathrm{a}=-0.601$ and $\mathrm{b}=-0.375$;

if SCreat $>0.7$ and CysC >0.8: $a=-0.601$ and $b=-0.711$ )

In males:

$\mathrm{eGFR}=130 \times(\text { SCreat } / 0.9)^{\mathrm{a}} \times(\text { CysC/0.8 })^{\mathrm{b}} \times 0.995^{\text {Age }}[\times 1.08$ if black]

(if SCreat $\leq 0.9$ and CysC $\leq 0.8: \mathrm{a}=-0.207$ and $\mathrm{b}=-0.375$;

if SCreat $\leq 0.9$ and CysC $>0.8: a=-0.207$ and $b=-0.711$;

if SCreat >0.9 and CysC $\leq 0.8: \mathrm{a}=-0.601$ and $\mathrm{b}=-0.375$;

if SCreat $>0.9$ and CysC $>0.8: a=-0.601$ and $b=-0.711$ )

SCreat is expressed in milligrams per deciliter, CysC in milligrams per liter and age in years.

Glomerular Filtration Rate Measurement

Children with bladder dysfunction, unable to void spontaneously, and in whom bladder catheterization failed, were excluded. Inulin clearance (iGFR) was obtained as follows: two intravenous catheters were inserted on admission, one in each arm and a loading dose of inulin $25 \%$ was administrated according to the 
(Inutest SPC; Fresenius Kabi Pharma Austria GmbH, Graz, Austria) protocol. The loading dose was calculated in order to obtain a required plasma concentration of $200-250 \mathrm{mg} / \mathrm{l}$, as follows: Loading inulin dose $=$ Required plasma concentration $\times$ Estimated inulin distribution volume. The estimated inulin distribution volume corresponds to the extracellular volume and amounts to $15 \%$ of the body weight (BW). Subsequently, inulin was continuously infused over $90 \mathrm{~min}$ at a rate given by the required inulin plasma concentration $(200-250 \mathrm{mg} / \mathrm{l})$ and the eGFR as follows: Infusion rate in $\mathrm{mg} / \mathrm{min}=$ (Required plasma concentration $/ 1,000) \times \mathrm{eGFR}$ in $\mathrm{ml} / \mathrm{min}$. Water diuresis was induced by oral administration of $20 \mathrm{ml} / \mathrm{kg}$ of water (maximum $1,200 \mathrm{ml}$ ) in the first hour followed by $3 \mathrm{ml} / \mathrm{kg} / \mathrm{h}$ of water. This was combined with an intravenous infusion of $0.9 \%$ sodium chloride (max. $300 \mathrm{ml}$ ) every $30 \mathrm{~min}$. After a 90-min equilibration period, three timed-urine samples were collected every $30 \mathrm{~min}$, according to the manufacturer's protocol (Inutest SPC; Fresenius Kabi Pharma Austria $\mathrm{GmbH}$ ), with a blood test in the middle of each urine collection. Inulin was measured using the anthrone method of Wright and Gann [12], using an Autoanalyzer 3 system (high-resolution digital colorimeter of SEAL; Bran + Luebbe, Norderstedt, Germany). The method was calibrated with five point calibrations (targets values at 10, 20, 30, 40, and $50 \mathrm{mg} / 100 \mathrm{ml}$ ) with coefficient correlations of $0.9993 \pm$ 0.0005 . The procedure is automatized with software (AACE 6.03; Bran + Luebbe) which automatically includes corrections for baseline, carryover, sensitivity drift and dilution factor. For the serum, the intra-assay CVs obtained in our laboratory with the internal quality controls were $2.44 \%$ at $10 \mathrm{mg} / 100 \mathrm{ml}, 1.47 \%$ at $30 \mathrm{mg} / 100 \mathrm{ml}$, and $0.94 \%$ at $40 \mathrm{mg} / 100 \mathrm{ml}$, while for urine the intra-assay CVs were $1.71 \%$ at $10 \mathrm{mg} / 100 \mathrm{ml}, 1.22 \%$ at $30 \mathrm{mg} /$ $100 \mathrm{ml}$, and $1.07 \%$ at $50 \mathrm{mg} / 100 \mathrm{ml}$. The inter-assay CVs obtained in the internal laboratory standards were $2.35 \%$ at $10 \mathrm{mg} / 100 \mathrm{ml}$, $2.23 \%$ at $30 \mathrm{mg} / 100 \mathrm{ml}$, and $0.87 \%$ at $50 \mathrm{mg} / 100 \mathrm{ml}$.

\section{Statistical Analysis}

Statistical analysis was performed using $\mathrm{R}$ software, version 2.13.1 (R Foundation for Statistical Computing, Vienna, Austria). Population demographics were reported as medians with upper and lower quartiles for continuous parameters and categorical variables were expressed as percentages.

The medians and 95\% CI of bias (iGFR-eGFR), the precision, i.e. the difference between lower and upper quartiles (interquartile range, IQR) of bias with the $95 \% \mathrm{CI}$ and the accuracies, i.e. the percentage of eGFR values that were within $30 \%$ of the iGFR values, were calculated for all formulas in the entire cohort, by CKD stages, and by age groups. The CKD-EPI and the Schwartz formulas performances were compared using the Wilcoxon signed-rank test. Confidence intervals were calculated by bootstrap (1,000 replicates). Significance was defined as $\mathrm{p}<0.05$.

\section{Results}

Medians with the lower and upper quartiles for age, BW, and Ht were 13.0 [8.9; 15.6] years, 41.9 [27.6; 57.5] $\mathrm{kg}$, and 150.1 [131.5; 166.5$] \mathrm{cm}$, respectively. $53 \%$ are males. Medians with the lower and upper quartiles for

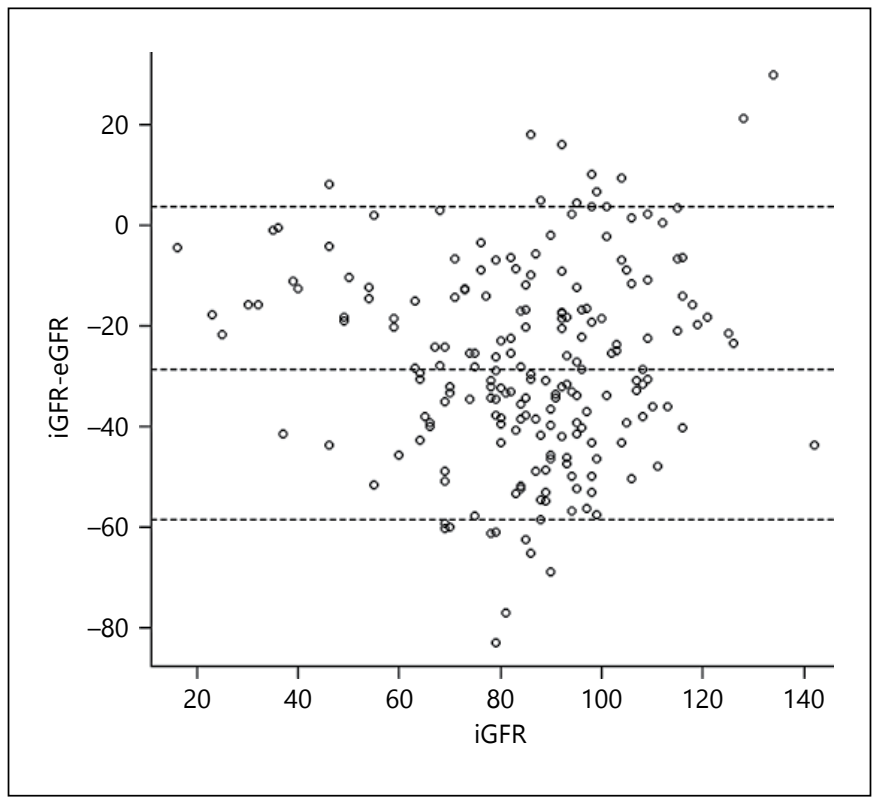

Fig. 1. Bias (difference between iGFR and eGFR) according to iGFR, using the combined CKD-EPI formula.

iGFRs and eGFRs using the combined CKD-EPI formula, the revised Schwartz formula and the combined Schwartz formula, were 87 [75; 98], 115 [95.8; 135.8], $91.6[76.5 ; 106]$, and $81[68 ; 89] \mathrm{ml} / \mathrm{min} / 1.73 \mathrm{~m}^{2}$, respectively.

For the entire cohort, medians and 95\% CI of bias were significantly higher $(\mathrm{p}<0.001)$ when using the CKD-EPI formula compared to the revised SCreat Schwartz formula and to the combined Schwartz formula, with values of $28.6[25.4 ; 31.6], 6.1[4.1 ; 7.8]$ and $-6[-4 ; 8] \mathrm{ml} / \mathrm{min} / 1.73$ $\mathrm{m}^{2}$, respectively. Medians with $95 \%$ CI of bias for eGFRs using the CKD-EPI, the revised SCreat Schwartz, and the combined Schwartz formulas versus iGFRs are represented in figures 1,2 and 3, respectively. They show the clear overestimation of iGFRs using the combined CKD-EPI formula (fig. 1) and also the outperformance of the revised SCreat and combined Schwartz formulas (fig. 2, 3) compared to the CKD-EPI formula for eGFRs in all patients. Precision when applying the CKD-EPI formula was significantly lower $(\mathrm{p}<0.001)$ than when using the revised SCreat and the combined Schwartz formulas, with values of 26.2 [22.9; 30.0], 16.9 [14.0; 19.5], and 16 $[14 ; 18] \mathrm{ml} / \mathrm{min} / 1.73 \mathrm{~m}^{2}$, respectively. For an accuracy of $30 \%$, only $42.3 \%$ of eGFRs were accurate when using the combined CKD-EPI formula compared to 88.1 and $96.5 \%$ for the revised SCreat and the combined Schwartz formulas, respectively. 


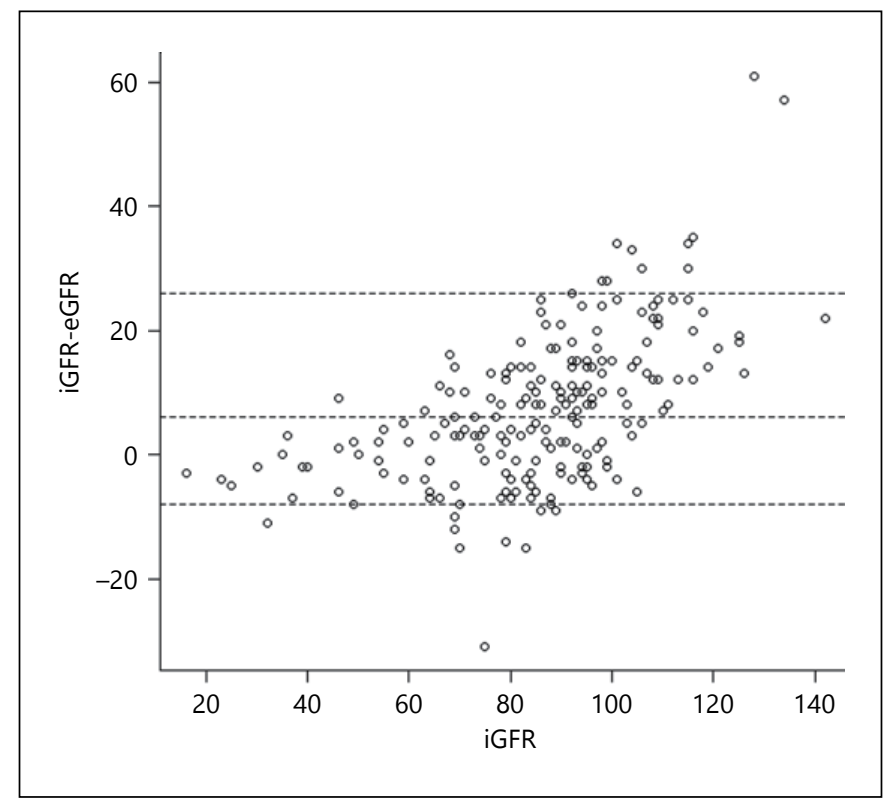

Fig. 2. Bias (difference between iGFR and eGFR) according to iGFR, using the revised SCreat Schwartz formula.

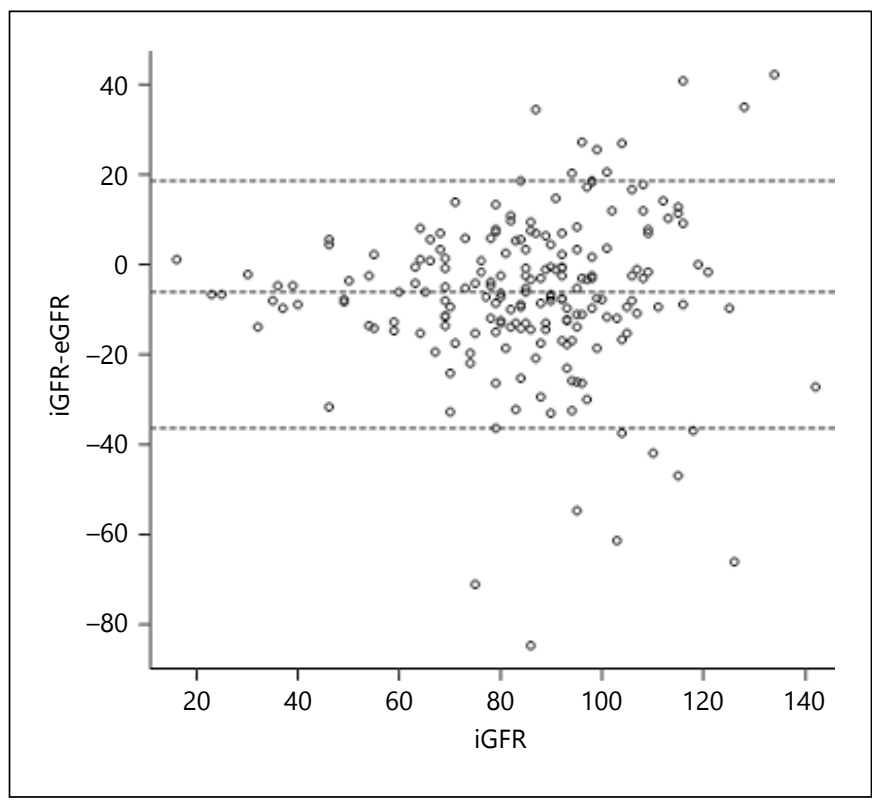

Fig. 3. Bias (difference between iGFR and eGFR) according to iGFR, using the combined Schwartz formula.

Table 2. Performances of the combined CKD-EPI formula, the solely SCreat-based Schwartz formula, and the combined SCreat and CysC Schwartz formula in terms of bias, precision (difference between lower and upper quartiles of bias), and accuracy at $30 \%$ for the entire cohort and for all CKD stages

\begin{tabular}{lcccc}
\hline & Entire cohort & CKD stage I & CKD stage II & CKD stages III-V \\
\hline Number of patients & 201 & 92 & 87 & 22 \\
Medians of bias using the combined CKD-EPI formula & $-28.6[-31.6 ;-25.4]$ & $-25.7[-31.8 ;-21.5]$ & $-33.3[-35.5 ;-30.5]$ & $-15.2[-18.4 ;-11.2]$ \\
Medians of bias using the SCreat Schwartz formula & $6.1[4.1 ; 7.8]$ & $-4.2[-7.9 ;-1.6]$ & $-6.1[-8.6 ;-4.1]$ & $-7.2[-8.9 ;-4.7]$ \\
Medians of bias using the combined Schwartz formula & $6[4 ; 8]$ & $13[10 ; 15]$ & $3[1 ; 4]$ & $-2[-3 ; 0.5]$ \\
Precision using the combined CKD-EPI formula & $26.2[22.9 ; 30.0]$ & $27.8[21.4 ; 34.3]$ & $20.9[15.2 ; 31.9]$ & $12.9[6.4 ; 26.0]$ \\
Precision using the SCreat Schwartz formula & $16.9[14.0 ; 19.5]$ & $20.5[15.1 ; 27.2]$ & $15.9[12.8 ; 20.0]$ & $9.3[4.7 ; 13.1]$ \\
Precision using the combined Schwartz formula & $16[14 ; 18]$ & $15.5[11.5 ; 19]$ & $15.5[12 ; 17]$ & $6[3 ; 8]$ \\
Accuracy using the combined CKD-EPI formula & $42.3[37.3 ; 48.3]$ & $57.6[48.9 ; 66.3]$ & $25.3[17.2 ; 33.3]$ & $45.4[27.3 ; 63.6]$ \\
Accuracy using the SCreat Schwartz formula & $88.1[84.1 ; 91.5]$ & $85.9[73.3 ; 91.3]$ & $89.7[83.9 ; 94.3]$ & $90.9[81.8 ; 100]$ \\
Accuracy using the combined Schwartz formula & $96.5[94.0 ; 98.5]$ & $94.6[90.2 ; 97.8]$ & $98.9[96.5 ; 100]$ & $95.5[86.4 ; 100]$ \\
\hline
\end{tabular}

For children in group 1 (CKD stage I), group 2 (CKD stage II) and group 3 (CKD stages III-V), medians and $95 \%$ CI of bias were significantly higher $(\mathrm{p}<0.001)$ when using the CKD-EPI formula compared to the both revised SCreat and combined Schwartz formulas (table 2). Precision when applying the CKD-EPI formula was significantly lower $(\mathrm{p}<0.001)$ than when using the revised SCreat or the combined Schwartz formulas (table 2). We also observed that the combined CKD-EPI formula precision increased with worsening of kidney function (ta-

Combined CKD-EPI Formula for

Estimating GFR in Children ble 2). Regarding accuracies, we observed that the combined CKD-EPI formula achieves its best accuracy of $57.6 \%$ in patients with CKD stage I.

When analyzing the performance of the combined CKD-EPI formula in relation to the ages of children, figure 4 shows that formula bias decreased while age increased. We therefore divided our population cohort into three age groups: group 1 includes children under the age of 10 years, group 2 includes children between the age of 10 and 15 years, and group 3 includes children 
Table 3. Performances of the combined CKD-EPI formula, the solely SCreat-based Schwartz formula, and the combined SCreat and CysC Schwartz formula in terms of bias and accuracy at $30 \%$ for children below the age of 10 years, between the age of 10 and 15 years and above the age of 15 years

\begin{tabular}{lccc}
\hline & Children $<10$ years & Children $10-15$ years & Children $>15$ years \\
\hline Children, $n$ & 65 & 76 & 60 \\
Medians of bias using the combined CKD-EPI formula & $-41.5[-45.8 ;-36.1]$ & $-25.5[-30.6 ;-20.1]$ & $-18.2[-22.5 ;-14.1]$ \\
Medians of bias using the SCreat Schwartz formula & $-10.0[-14.2 ;-8.6]$ & $-5.2[-8.6 ;-2.4]$ & $-0.7[-3.4 ; 3.4]$ \\
Medians of bias using the combined Schwartz formula & $7[3 ; 10]$ & $8[4.5 ; 10]$ & $3[0 ; 7]$ \\
Accuracy using the combined CKD-EPI formula & $21.5[13.8 ; 29.2]$ & $48.7[39.5 ; 57.9]$ & $57.6[47.5 ; 67.8]$ \\
Accuracy using the SCreat Schwartz formula & $76.9[67.7 ; 84.6]$ & $94.7[90.8 ; 98.7]$ & $93.2[86.4 ; 98.3]$ \\
Accuracy using the combined Schwartz formula & $96.9[92.3 ; 100]$ & $100[100 ; 100]$ & $91.7[85.0 ; 96.7]$ \\
\hline
\end{tabular}

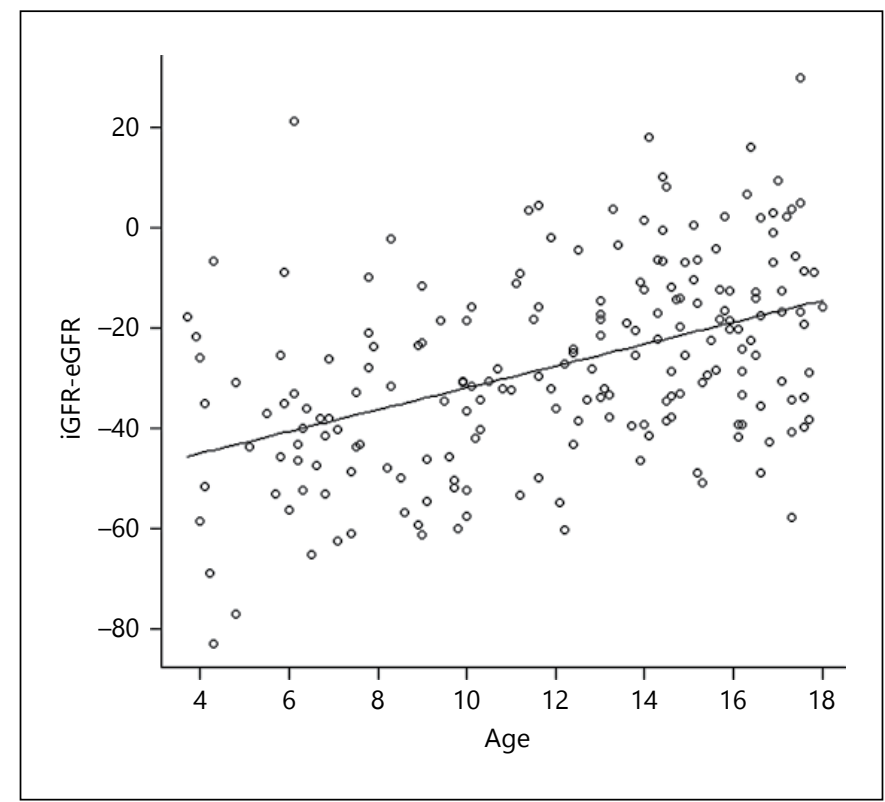

Fig. 4. Performance of the combined CKD-EPI formula in relation to the ages of children showing that formula bias decreased while age increased.

above the age of 15 years. The CKD-EPI formula bias decreased and accuracy increased with the increasing of child age group, and a better formula performance (lower bias, greater accuracy) was observed in children above 15 years of age (group 3). However, medians of bias of the CKD-EPI formula was significantly $(\mathrm{p}<0.001)$ greater and accuracy lower than the revised SCreat and the combined Schwartz formulas across all children's age groups (table 3). Medians with 95\% CI of bias and accuracies of each formula in the different age groups are presented in table 3 .

\section{Discussion}

SCreat was the first endogenous marker used for deriving eGFR formulas $[13,14]$, however SCreat measurement is significantly influenced by muscle mass, age, gender, diet and tubular secretion [15], and consequently these formulas are far from ideal to estimate GFR. Therefore, in order to compensate for the inaccuracy of the solely SCreat-based formulas, alternative formulas based on combined SCreat and CysC as an endogenous marker that seems to be independent of age, body weight or height [16] have been derived.

Recognizing a universal formula applicable for both adult and children groups is challenging and several studies evaluating the reliability of adults' formulas in a pediatric population have been performed using the Cockcroft-Gault [13], the Modification of Diet in Renal Disease (MDRD) [5], and the SCreat-based CKD-EPI [6] formulas. Pierrat et al. [17] found that the CockcroftGault formula is appropriate for estimating GFR in children over the age of 12 years and that the MDRD formula presents a large overestimation in all children above 3 years of age. However, Filler et al. [18] concluded that the Cockcroft-Gault formula poorly estimates GFR in children with a considerable bias. On the other hand, Selistre et al. [19] and Chehade et al. [20] recommended against the use of the Cockcroft-Gault, MDRD, and SCreat-based CKD-EPI formulas in children. More recently, Levey and co-workers [7] developed a new CKDEPI combined formula, applicable in adults population, that provides the most accurate estimate of GFR compared to the previously known SCreat-based CKD-EPI formula and also to the CysC-based CKD-EPI formula. They state that this new combined CKD-EPI formula represents an advance over the currently available adults' 
equations across all GFR levels. However, the applicability of this new combined formula in pediatric population is confronted with several limitations. We have demonstrated that this new formula, compared to the most widely used formula in children, i.e. the revised SCreat Schwartz formula [8], and also to the combined SCreat and CysC Schwartz formula [10], shows a poor performance across all GFR levels and in each CKD groups. In analogy to adult CKD groups, accuracy of this new formula reaches its best in children with less renal impairment (stage I CKD) compared to children and adults with moderate to severe renal failure. We also observe that the new CKD-EPI formula bias decreases as the age increases, with a better accuracy in the adolescent age group. Nevertheless, formulas bias and errors remain important and accuracies values are still low to be validated, with a significant difference when compared to both solely SCreat and combined SCreat and CysC Schwartz formulas performance for eGFR in this adolescent age group. These results lead to conclude that there are other factors not included in the CKD-EPI equation that significantly affects formula accuracies and results, making it non-applicable in pediatric population groups. One of these factors is related to the fact that in pediatric age group, muscle mass increases with age and is - in fact - more closely correlated with height than age (which is the concept underlying the Schwartz equations). In contrast, in adults, muscle mass decreases with time and therefore in the new CKD-EPI equation, age was introduced as an exponential factor (i.e. $0.995^{\text {age }}$ ). This yields when applying the new CKD-EPI formula, to a higher eGFR in a young compared to an older subject with equal creatinine levels.
The strength of this study is the use of a large database of 201 measured GFRs with inulin clearances that are recognized as the gold standard method of measuring GFR. It is also, to our knowledge, the first report that evaluates the new combined CKD-EPI formula in children and demonstrates that its performance improves in adolescence.

This study has several limitations. SCreat measurements were performed using the compensated Jaffe technique, therefore we cannot rule out the fact that we may have underestimated the performance of the combined CKD-EPI formula, secondary to the different SCreat measurements method used in the CKD-EPI cohort (enzymatic method). This study did not allow determining factors that affect the new CKD-EPI formula performance in children and therefore we recommend further research in this field. Finally, our cohort included only 2 black children, therefore, we cannot draw any conclusion for this group.

In conclusion, despite the advance in the performance of the new combined CKD-EPI formula for eGFR in the adult population, it is still unreliable in children due to the differences in non-GFR determinants of creatinine during growth and development and cannot therefore be applied in this population across all CKD stages and all child ages.

\section{Disclosure Statement}

The authors have no conflicts of interest to disclose.

\section{References}

1 Vassalotti JA, Stevens LA, Levey AS: Testing for chronic kidney disease: a position statement from the National Kidney Foundation. Am J Kidney Dis 2007;50:169-180.

2 Madero M, Sarnak MJ: Creatinine-based formulae for estimating glomerular filtration rate: is it time to change to chronic kidney disease epidemiology collaboration equation? Curr Opin Nephrol Hypertens 2011;20:622630.

3 Sharma AP, Yasin A, Garg AX, Filler G: Diagnostic accuracy of cystatin C-based eGFR equations at different GFR levels in children. Clin J Am Soc Nephrol 2011;6:1599-1608.

4 Stevens LA, Levey AS: Measured GFR as a confirmatory test for estimated GFR. J Am Soc Nephrol 2009;20:2305-2313.
5 Levey AS, Greene T, Kusek JW, Beck GJ: A simplified equation to predict glomerular filtration rate from serum creatinine. J Am Soc Nephrol 2000;11:A0828.

6 Levey AS, Stevens LA, Schmid CH, Zhang YL, Castro AF 3rd, Feldman HI, Kusek JW, Eggers P, Van Lente F, Greene T, Coresh J; CKDEPI (Chronic Kidney Disease Epidemiology Collaboration): A new equation to estimate glomerular filtration rate. Ann Intern Med 2009; 150:604-612.

7 Inker LA, Schmid CH, Tighiouart H, Eckfeldt JH, Feldman HI, Greene T, Kusek JW, Manzi J, Van Lente F, Zhang YL, Coresh J, Levey AS, CKD-EPI Investigators: Estimating glomerular filtration rate from serum creatinine and cystatin C. N Engl J Med 2012;367:20-29.
8 Schwartz GJ, Muñoz A, Schneider MF, Mak RH, Kaskel F, Warady BA, Furth SL: New equations to estimate GFR in children with CKD. J Am Soc Nephrol 2009;20:629-637.

9 Gao A, Cachat F, Faouzi M, Bardy D, Mosig D, Meyrat BJ, Girardin E, Chehade H: Comparison of the glomerular filtration rate in children by the new revised Schwartz formula and a new generalized formula. Kidney Int 2013;83:524-530.

10 Schwartz GJ, Schneider MF, Maier PS, Moxey-Mims M, Dharnidharka VR, Warady BA, Furth SL, Muñoz A: Improved equations estimating GFR in children with chronic kidney disease using an immunonephelometric determination of cystatin C. Kidney Int 2012;82: 445-453.
Combined CKD-EPI Formula for

Estimating GFR in Children
Am J Nephrol 2013;38:300-306 DOI: $10.1159 / 000354920$ 
11 KDIGO CKD Work Group: KDIGO clinical practice guideline for the evaluation and management of chronic kidney disease. Kidney Int 2013;3(suppl):1-150.

12 Wright HK, Gann DS: An automatic anthrone method for determination of inulin in plasma and urine. J Lab Clin Med 1966;67: 689-693.

13 Cockroft DW, Gault MH: Prediction of creatinine clearance from serum creatinine. Nephron 1976;16:31-41.

14 Schwartz GJ, Haycock GB, Edelmann CM Jr, Spitzer A: A simple estimate of glomerular filtration rate in children derived from body length and plasma creatinine. Pediatrics 1976; 58:259-263.
15 Stevens LA, Coresh J, Greene T, Levey AS: Assessing kidney function: measured and estimated glomerular filtration rate. $\mathrm{N}$ Engl $\mathrm{J}$ Med 2006;354:2473-2483.

16 Andersen TB, Eskild-Jensen A, Frokiaer J, Brochner-Mortensen J: Measuring glomerular filtration rate in children: can cystatin $\mathrm{C}$ replace established methods? A review. Pediatr Nephrol 2009;24:929-941.

17 Pierrat A, Gravier E, Saunders C, Caira MV, Aït-Djafer Z, Legras B, Mallié JP: Predicting GFR in children and adults: a comparison of the Cockcroft-Gault, Schwartz, and Modification of Diet in Renal Disease formulas. Kidney Int 2003;64:1425-1436.
18 Filler G, Foster J, Acker A, Lepage N, Akbari A, Ehrich JH: The Cockcroft-Gault formula should not be used in children. Kidney Int 2005;67:2321-2324.

19 Selistre L, De Souza V, Cochat P, Antonello IC, Hadj-Aissa A, Ranchin B, Dolomanova $\mathrm{O}$, Varennes A, Beyerle F, Bacchetta J, Dubourg L: GFR estimation in adolescents and young adults. J Am Soc Nephrol 2012;23: 989-996.

20 Chehade H, Girardin E, Iglesias K, Ramseyer P, Frey P, Bardy D, Mosig D, Cachat F: Assessment of adult formulas for glomerular filtration rate estimation in children. Pediatr Nephrol 2013;28:105-114. 